\title{
Combined Modality Therapy for Rectal Cancer: Too Much Too Often?
}

\author{
Martin R. Weiser, MD \\ Colorectal Service, Department of Surgery, Memorial Sloan-Kettering Cancer Center, New York, NY
}

The refinement and implementation of multimodal therapy for rectal cancer, including surgery, radiotherapy, and chemotherapy, is certainly one of the most meaningful achievements in the history of clinical cancer research to date. It is thus important to set any harsh critique of current treatment methods and outcomes within this context.

Just 30 years ago, the risk of local recurrence after rectal resection ranged from 20 to $40 \%{ }^{1,2}$ It was often lethal and was associated with significant morbidity, pain, and suffering. ${ }^{3,4}$ This began to change with the report from the National Surgical Adjuvant Breast and Bowel Project (NSABP) Cooperative Group R01 trial in 1985, which demonstrated a reduction in local recurrence from 25 to $16 \%$ with the addition of postoperative long-course chemoradiotherapy. ${ }^{1}$

The NSABP trial was followed by serial studies designed to improve results through addition and resequencing. The Gastrointestinal Tumor Study Group (GITSG) reported a 55-33\% reduction in all recurrence with the addition of adjuvant chemotherapy and radiotherapy, leading the National Institutes of Health to generate a consensus statement asserting that chemoradiotherapy should be delivered postoperatively for all stage II and III tumors. ${ }^{2}$ The German Rectal Cancer Study later demonstrated improved outcome when long-course chemoradiotherapy was delivered preoperatively rather than postoperatively to clinical stage II and III rectal cancers. Local recurrence at 46 months was reduced from 13 to $6 \%$ without change in distant recurrence. At 10 years, the difference in local recurrence was of a lower magnitude but remained significant (7 vs. $10 \%$ ). Disease-free and overall survival did not differ between the groups. ${ }^{5}$ This changed the standard of practice to preoperative rather than

(C) Society of Surgical Oncology 2013

Published Online: 19 March 2013

M. R. Weiser, MD

e-mail: weiser1@mskcc.org postoperative long-course chemoradiotherapy for stage II and III rectal cancers.

While North America and Central Europe employed a regimen of long-course postoperative chemoradiotherapy administered over 5-6 weeks, Northern Europe was utilizing short-course radiotherapy delivered over 5 days before surgery, and demonstrating similar improvements in outcome. The Swedish Rectal Cancer Trial showed that preoperative short-course radiotherapy reduced local recurrence from 27 to $11 \%$ and increased survival from 65 to $74 \%$ compared with surgery alone. ${ }^{6}$

This brings us to 2013, when patients are much less likely to experience local recurrence and more likely to retain sphincter function than decades ago. However, two reports in this issue of Annals of Surgical Oncology remind us that more is not necessarily better, and that one size does not fit all. Lange et al. report on a series of rectal cancer patients treated traditionally under the supervision of Warren Enker, an international leader and pioneer who has spent his career spreading the word about the methodical techniques of anatomic rectal resection and the benefits of total mesorectal excision. ${ }^{7}$ In total, 368 patients underwent rectal resection, with a median follow-up of 8 years. Seventy percent underwent sphincter-preserving surgery, with an average anastomotic height of $5.4 \mathrm{~cm}$. Only $5 \%$ of patients undergoing low anterior resection experienced anastomotic leak. The oncologic results are equally impressive. Five-year overall survival was $76 \%$ : $92 \%$ for stage I disease, $78 \%$ for stage II disease, and $69 \%$ for stage III disease. The 5-year local recurrence rate was $5.2 \%: 0.8 \%$ for stage I disease, $4.7 \%$ for stage II disease, and $5.4 \%$ for stage III disease. The 5-year rate of distant metastasis was $22 \%: 3.9 \%$ for stage I disease, $21 \%$ for stage II disease, and $27 \%$ for stage III disease.

Analysis of functional outcome was measured in 50 patients. Irradiated patients had, on average, 3.2 bowel movements per day, compared to 1.2 in nonirradiated 
patients. Twenty-one of 25 irradiated patients had bowel movements during the night, compared to 3 of 13 nonirradiated patients. Of more concern was the observation that long-term fecal incontinence was experienced by 20 of 25 irradiated patients, compared to only 1 of 13 nonirradiated patients. Radiation remained an independent risk factor for poor bowel function after adjusting for anastomotic height, gender, and age. In other words, surgery remains the key intervention, and additional therapy is not without significant cost.

Also in this issue of Annals of Surgical Oncology, Loos et al. report a systematic review and meta-analysis of the impact of preoperative neoadjuvant radio(chemo)therapy on long-term functional outcomes in resectable rectal cancer. ${ }^{8}$ They investigated anorectal, sexual, and urinary function in a collection of 25 studies including 6,548 patients. Although methodological quality was low, the majority of studies reported on anorectal function. Metaanalysis revealed that stool incontinence occurred 1.67 times more often in irradiated patients. These findings were corroborated by the manometric data, which demonstrated lower resting and squeeze pressures in irradiated patients.

Taken together, these two reports clearly point out the downside of multimodality treatment and raise important questions: Are all stage II and III rectal cancer patients at high risk for local recurrence, and do all of them require such exhaustive treatment? Can treatment be individualized, or tailored, on the basis of a specific oncologic evaluation of each patient - an evaluation that will identify those who may not need to undergo certain elements of standard neoadjuvant therapy?

Chemoradiotherapy was traditionally thought to reduce pelvic recurrence by sterilizing any disease remaining after rectal resection. However, until the 1980s the pervasive surgical technique included blunt posterior dissection, which often led to incomplete mesorectal excision or presacral bleeding due to tearing of the endopelvic fascia. In 1982, Heald published his landmark article describing outcomes after total mesorectal excision (TME), a technique defined by sharp dissection under direct visualization of the visceral and parietal layers of the endopelvic fascia. ${ }^{9}$ The result was a specimen with an intact mesorectum and negative tumor margins in the majority of resectable rectal cancers. Heald's first series of 122 curative anterior resections reported an astounding $2.7 \%$ rate of local recurrence at 5 years and $88 \%$ overall survival. ${ }^{10}$ These excellent results were subsequently matched in a series by Enker et al. ${ }^{11}$ which included 246 curable Dukes stage B and $\mathrm{C}$ patients. Only 18 developed local recurrence, and the actuarial cancer-specific 5-year survival was $74 \%$. Enker et al. ${ }^{11,12}$ championed this technique of anatomic resection, demonstrating improved autonomic nerve preservation and less postoperative sexual and bladder dysfunction.
The Dutch Colorectal Cancer Group attempted to determine whether radiotherapy was necessary when surgery was performed using the techniques of TME. After formal instruction, surgeons performed TME with or without preoperative short-course radiotherapy. The results showed a benefit of radiotherapy even in the setting of TME, with an 11-5\% reduction in local recurrence at 10 years but no change in overall survival. Interpretation of these results is limited, however, as $24 \%$ of TME specimens in this study were found to be suboptimal on pathologic review. ${ }^{13}$

Concerns that radiation to the pelvis was associated with long-term toxicity were substantiated in follow-up studies reported by the Dutch Colorectal Cancer Group. Although bowel function improved in the first 1-2 years after surgery, a slow but steady decline was noted over the subsequent 3 years. ${ }^{14}$ In patients who survived 5 years, incontinence was reported in $68 \%$ of the irradiated group, compared to $38 \%$ of the nonirradiated group. ${ }^{15}$

The reports by Loos et al. and Lange et al. in this issue of Annals of Surgical Oncology expand on these findings, adding to our growing apprehension about use of chemoradiotherapy for all stage II and III rectal cancer patients. The fact that the NSABP investigators did not think of the possible biological differences between tumors when they began their first rectal cancer study is excusable in that oncogenes had not yet been discovered at the time. Now that we know better, it appears self-evident that we should not be treating all stage II and III rectal cancer patients as a homogeneous group.

Gunderson attempted to resolve this using a pooled analysis of prospective and randomized datasets. He was able to develop risk groups based on local recurrence. Stage I represented a low-risk group, as expected. Interestingly, an intermediate-risk group was identified, characterized by tumor, node, metastasis classification system (TNM) stage T1-2N1 and T3N0 lesions, with a moderate $(6-8 \%)$ risk of local recurrence. ${ }^{16-18}$ Clearly there is a subset of stage II and III patients who may not require intensive trimodal therapy.

There are clinical data to suggest that selective use of multimodality therapy is indeed rational. Cercek et al. ${ }^{19}$ reported a small retrospective series of 14 colorectal cancer patients treated with chemotherapy alone in the setting of stage IV disease or other contraindication for pelvic radiation. In this study, 5 of 14 patients experienced a complete pathologic response; an additional 4 showed greater than $80 \%$ tumor destruction. In the subset of 7 rectal cancer patients, 3 experienced a complete pathologic response; an additional 3 showed greater than $90 \%$ tumor destruction.

The hypothesis that primary rectal cancer is sensitive to modern systemic chemotherapy, and that radiotherapy can be used selectively, was then prospectively studied in 32 
stage II and III rectal cancer patients. All 30 patients who completed neoadjuvant chemotherapy had clinical responses and underwent complete R0 resection. Eight patients $(27 \%)$ had a complete pathologic response, and a total of $19(63 \%)$ showed greater than $80 \%$ tumor destruction at time of resection. Three patients experienced recurrence with pulmonary metastases. At a mean followup of 27 months, no study participant had a pelvic recurrence. ${ }^{20}$

These promising results have led to an international phase II/III prospective randomized trial attempting to individualize treatment of rectal cancer. The PROSPECT (N1048) Trial (Preoperative Radiation Or Selective Preoperative radiation and Evaluation before Chemotherapy and Total mesorectal excision) asks whether pelvic radiotherapy can be used selectively rather than uniformly. Rectal cancer patients with TNM stage T3N0, T3N1, and $\mathrm{T} 2 \mathrm{~N} 1$ disease are treated with preoperative chemotherapy, and the disease is then restaged. If a significant response is observed, patients continue on to definitive rectal surgery. If there has not been a significant response, patients proceed with standard chemoradiotherapy, followed by surgery. Appropriate correlative science studies will shed more light on the biology of rectal cancer, while innovative quality-of-life analyses will further define treatment-related toxicity and its postoperative impact on patients.

In summary, we have made tremendous strides in the treatment of rectal cancer, and we owe a debt of gratitude to the preceding generation of surgeons and oncologists who envisioned a way to improve patient outcomes. Because of these studies, mortality is most commonly related to distant metastasis rather than local recurrence. While maintaining excellence in surgical techniques, we must concentrate our efforts on new therapies that will reduce distant relapse while limiting treatment-related toxicity and retaining a high quality of life for our patients. The reports by Loos et al. and Lange et al. highlight the importance of questioning conventional treatment paradigms. Bold studies such as PROSPECT are a necessary first step in the individualization of treatment based on risk. This will advance our understanding of rectal cancer biology, and will ultimately propel the field forward.

DISCLOSURE The author declares no conflict of interest.

\section{REFERENCES}

1. Farias EF, Ong DE, Ghyselinck NB, Nakajo S, Kuppumbatti YS, Mira y Lopez R. Cellular retinol-binding protein I, a regulator of breast epithelial retinoic acid receptor activity, cell differentiation, and tumorigenicity. J Natl Cancer Inst. 2005;97:21-9.
2. Gastrointestinal Tumor Study Group. Prolongation of the diseasefree interval in surgically treated rectal carcinoma. $N$ Engl J Med. 1985;312:1465-72.

3. Cohen AM, Minsky BD. Aggressive surgical management of locally advanced primary and recurrent rectal cancer. Current status and future directions. Dis Colon Rectum. 1990;33:432-8.

4. Salo JC, Paty PB, Guillem J, Minsky BD, Harrison LB, Cohen AM. Surgical salvage of recurrent rectal carcinoma after curative resection: a 10-year experience. Ann Surg Oncol. 1999;6:171-7.

5. Sauer R, Liersch T, Merkel S, et al. Preoperative versus postoperative chemoradiotherapy for locally advanced rectal cancer: results of the German CAO/ARO/AIO-94 randomized phase III trial after a median follow-up of 11 years. J Clin Oncol. 2012;30:1926-33.

6. Improved survival with preoperative radiotherapy in resectable rectal cancer. Swedish Rectal Cancer Trial. $N$ Engl J Med. 1997;336:980-7.

7. Lange M, et al. Long term results of rectal cancer surgery with a systematical operative approach. Ann Surg Oncol. doi: 10.1007/s10350-008-9328-y.

8. Loos M, et al. Effect of preoperative radio(chemo)therapy on long-term functional outcome in rectal cancer patients: a systematic review and meta-analysis. Ann Surg Oncol. doi: 10.1245/s10434-012-2827-z.

9. Heald RJ, Husband EM, Ryall RD. The mesorectum in rectal cancer surgery-the clue to pelvic recurrence? $\mathrm{Br} J$ Surg. 1982;69:613-6.

10. Heald RJ, Ryall RD. Recurrence and survival after total mesorectal excision for rectal cancer. Lancet. 1986;1(8496):1479-82.

11. Enker WE, Thaler HT, Cranor ML, Polyak T. Total mesorectal excision in the operative treatment of carcinoma of the rectum. J Am Coll Surg. 1995;181:335-46.

12. Enker WE. Potency, cure, and local control in the operative treatment of rectal cancer. Arch Surg. 1992;127:1396-401.

13. Nagtegaal ID, van de Velde CJ, van der Worp E, Kapiteijn E, Quirke P, van Krieken JH; Cooperative Clinical Investigators of the Dutch Colorectal Cancer Group. Macroscopic evaluation of rectal cancer resection specimen: clinical significance of the pathologist in quality control. J Clin Oncol. 2002;20:1729-34.

14. Lange MM, den Dulk M, Bossema ER, Cooperative Clinical Investigators of the Dutch Total Mesorectal Excision Trial, et al. Risk factors for faecal incontinence after rectal cancer treatment. Br J Surg. 2007;94:1278-84.

15. Peeters KC, van de Velde CJ, Leer JW, et al. Late side effects of short-course preoperative radiotherapy combined with total mesorectal excision for rectal cancer: increased bowel dysfunction in irradiated patients-a Dutch colorectal cancer group study. J Clin Oncol. 2005;23:6199-206.

16. Gunderson LL, Sargent DJ, Tepper JE, et al. Impact of T and N substage on survival and disease relapse in adjuvant rectal cancer: a pooled analysis. Int J Radiat Oncol Biol Phys. 2002;54:386-96.

17. Gunderson LL, Callister M, Marschke R, Young-Fadok T, Heppell J, Efron J. Stratification of rectal cancer stage for selection of postoperative chemoradiotherapy: current status. Gastrointest Cancer Res. 2008;2:25-33.

18. Gunderson LL, Sargent DJ, Tepper JE, et al. Impact of T and N stage and treatment on survival and relapse in adjuvant rectal cancer: a pooled analysis. J Clin Oncol. 2004;22:1785-96.

19. Cercek A, Weiser MR, Goodman KA, et al. Complete pathologic response in the primary of rectal or colon cancer treated with FOLFOX without radiation. J Clin Oncol. 2010;28(15 Suppl): 3649.

20. Schrag D, Weiser MR, Goodman KA, et al. Neoadjuvant FOLFOX-bev, without radiation, for locally advanced rectal cancer. J Clin Oncol. 2010;28(15 Suppl):3511. 